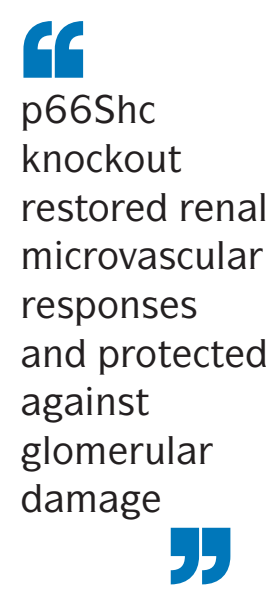

\title{
HYPERTENSION
}

\section{Role of p66Shc in renal vascular dysfunction}

A new study provides insights into the mechanisms by which regulation of renal microvascular tone is impaired in salt-sensitive hypertensioninduced nephropathy. Andrey Sorokin and colleagues report that overexpression of the $\mathrm{SH} 2$ adaptor protein p66Shc promotes renal vascular dysfunction in this disease.

p66Shc is known to have a role in the pathogenesis of age-related renal diseases and Sorokin and colleagues previously showed that the vasoactive peptide endothelin-1 (ET-1) signals through this protein. "In our recent study, discovery of the expression of p66Shc in the smooth muscle cells of renal resistance arteries from Dahl salt-sensitive rats maintained on a $1 \%$ salt diet prompted us to evaluate the role of p66Shc in regulation of renal vascular tone," explains Sorokin. They found that overexpression of p66Shc increased renal dysfunction in these rats, whereas p66Shc knockout restored renal microvascular responses and protected against glomerular damage.

To investigate the mechanism by which p66Shc impairs microvascular responses, the researchers used smooth muscle cells isolated from the renal interlobar arteries of genetically modified and wild type salt-sensitive rats. They showed that p66Shc knockout significantly increased ET-1-induced contraction of these cells. Moreover, they report that $\mathrm{p} 66 \mathrm{Shc}$ inhibits the activation of transient receptor potential canonical channels, thereby preventing the influx of calcium into smooth muscle cells that is required for ET-1-mediated regulation of vascular tone.

"Expression of p66Shc in the smooth muscle cells of renal afferent arterioles could cause these arteries to lose their ability to autoregulate vascular tone - this property is imperative for their task of preventing glomerular damage by maintaining the pressure gradients between renal capillaries and larger vessels," says Sorokin. "The uncovering of this new function of p66Shc expands our understanding of its role in renal pathophysiology and suggests that p66Shc is not just an 'ageing' gene."

Ellen F. Carney

ORIGINAL ARTICLE Miller, B. et al. p66Shc

regulates renal vascular tone in hypertensioninduced nephropahy.J. Clin. Invest. http://dx.doi.org/ $10.1172 / / \mathrm{Cl} 75079$ (2016) 